UCD-98-9

June 1998

hep-th/9806026

\title{
What We Don't Know about BTZ Black Hole Entropy
}

\author{
S. CARLIP \\ Department of Physics \\ University of California \\ Davis, CA 95616 \\ USA
}

\begin{abstract}
With the recent discovery that many aspects of black hole thermodynamics can be effectively reduced to problems in three spacetime dimensions, it has become increasingly important to understand the "statistical mechanics" of the (2+1)-dimensional black hole of Bañados, Teitelboim, and Zanelli (BTZ). Several conformal field theoretic derivations of the BTZ entropy exist, but none is completely satisfactory, and many questions remain open: there is no consensus as to what fields provide the relevant degrees of freedom or where these excitations live. In this paper, I review some of the unresolved problems and suggest avenues for their solution.
\end{abstract}

*email: carlip@dirac.ucdavis.edu 


\section{Introduction}

Since its discovery in 1992, the (2+1)-dimensional black hole of Bañados, Teitelboim, and Zanelli [1,2] has served as a useful model for realistic black hole physics [3]. Interest in this model has recently heightened with the discovery that the thermodynamics of higher-dimensional black holes can often be understood in terms of the BTZ solution. Many of the black holes relevant to string theory have near-horizon geometries of the form $B T Z \times M$, where $M$ is a simple manifold, and their entropies and grey-body factors can be obtained the BTZ black hole directly $44,5,6,6,8,8,9,10,11,12,13,14,15,16$, 17, 18, 19] or by duality [20,21,22]. It has become vital to understand BTZ black hole thermodynamics from first principles.

A natural guess is that black hole entropy should be associated with horizon degrees of freedom. In $2+1$ dimensions, the horizon is two-dimensional, suggesting the relevance of conformal field theory. This connection is strengthened by the observation that $(2+1)$ dimensional gravity can be written as a Chern-Simons theory [23, 24], and that ChernSimons theories induce Wess-Zumino-Witten models on boundaries [25, 26].

The first application of conformal field theory techniques to BTZ black hole entropy appeared in Ref. 27, with the treatment of boundary conditions later simplified in Ref. 28. This approach explicitly counts states, but relies on a poorly understood nonunitary theory and a complicated treatment of boundary data. It was recently shown that the same technique gives the correct entropy for $(2+1)$-dimensional de Sitter space [29]. A simpler computation, based on the Euclidean partition function, was developed in Ref. 30. Like most partition function methods, however, this approach does not explicitly display the states being counted, and it involves an analytic continuation from Lorentzian to Riemannian metrics that is not completely understood. A rather different Euclidean partition function approach appeared in Ref. 31 .

Recently, Strominger has suggested a much simpler derivation of the BTZ black hole entropy [4]. He begins with the observation, known since 1986 [32], that the asymptotic symmetry group of $(2+1)$-dimensional gravity with a negative cosmological constant $\Lambda=-1 / \ell^{2}$ is generated by two copies of the Virasoro algebra, with central charges

$$
c_{L}=c_{R}=\frac{3 \ell}{2 G}
$$

The degrees of freedom, now at infinity rather than the horizon, are thus described by a conformal field theory with this central charge. The asymptotic density of states for such a theory follows from a result of Cardy's [33, 34]: it is

$$
\ln \rho(\Delta, \bar{\Delta}) \sim 2 \pi \sqrt{\frac{c_{R} \Delta}{6}}+2 \pi \sqrt{\frac{c_{L} \bar{\Delta}}{6}},
$$


where $\Delta$ and $\bar{\Delta}$ are the eigenvalues of the two Virasoro generators $L_{0}$ and $\bar{L}_{0}$. But for the BTZ black hole, we have (up to an ambiguous additive constant) 35]

$$
M=\left(L_{0}+\bar{L}_{0}\right) / \ell, \quad J=L_{0}-\bar{L}_{0}
$$

where $r_{ \pm}$are the radii of the inner and outer horizons. Substituting into (11.2) and using the expression (A.4) for the mass and angular momentum, we obtain the correct entropy

$$
S=\frac{2 \pi r_{+}}{4 G}
$$

Attractive as this approach is, it is not yet the full answer. The Cardy formula (1.2) is derived from the partition function, and like the Euclidean approach of Ref. 30, it hides the actual degrees of freedom that contribute to the entropy. Moreover, as we shall see below, equation (1.2) involves some hidden assumptions that may not hold for the BTZ black hole. Strominger's derivation also raises the question of where the relevant degrees of freedom are located: other approaches describe excitations at the horizon, but the central charges (1.1) are relevant for a conformal field theory at infinity.

In this paper, I will discuss these issues, describing some of the assumptions and ambiguities in various approaches and suggesting a few paths forward. While I have tried to make this work reasonably self-contained, I assume some familiarity with the BTZ solution (see Ref. 3 for a review). An appendix describes some coordinate systems and conventions, and a second appendix discusses issues related to the choice of boundary conditions in Ref. 27.

It is perhaps worth emphasizing that although some of the approaches described here are inspired by string theory, my focus is on pure $(2+1)$-dimensional gravity. A full string theoretical picture of the BTZ black hole presumably involves a large number of added degrees of freedom (see, for example, [36]), and the relationship to the counting techniques described here is not entirely clear.

\section{Counting States: Partition Functions and Cardy's Formula}

In the microcanonical ensemble, the entropy is essentially the logarithm of the density of states $\rho(E)$. There are two common methods for determining this quantity. The most straightforward, which I address in section 3, is to simply count: we begin with a vacuum state and see how many different ways we can add excitations to reach the energy $E$. The second approach is less direct, but often simpler: we manipulate the partition function to obtain a density of states. For conformal field theories, this method yields the Cardy formula (1.2) and its generalization, equation (2.13) below. 
We begin with a conformal field theory with central charge $c$, with the standard Virasoro algebra

$$
\left[L_{m}, L_{n}\right]=(m-n) L_{m+n}+\frac{c}{12} m\left(m^{2}-1\right) \delta_{m+n, 0} .
$$

Cardy's basic result [33, 34] is that the quantity

$$
Z_{0}(\tau, \bar{\tau})=\operatorname{Tr} e^{2 \pi i\left(L_{0}-\frac{c}{24}\right) \tau} e^{-2 \pi i\left(\bar{L}_{0}-\frac{c}{24}\right) \bar{\tau}}
$$

is modular invariant, and in particular invariant under the transformation $\tau \rightarrow-1 / \tau$. This argument involves only some quite general properties of conformal field theories, and I will assume it holds for the theory associated with the BTZ black hole.

Now, the partition function on the torus of modulus $\tau$ is

$$
Z(\tau, \bar{\tau})=\operatorname{Tr} e^{2 \pi i \tau L_{0}} e^{-2 \pi i \bar{\tau} \bar{L}_{0}}=\sum \rho(\Delta, \bar{\Delta}) e^{2 \pi i \Delta \tau} e^{-2 \pi i \bar{\Delta} \bar{\tau}}
$$

For a unitary theory, $\rho$ is the number of states with eigenvalues $L_{0}=\Delta, \bar{L}_{0}=\bar{\Delta}$, as can be seen by inserting a complete set of states into the trace. For a nonunitary theory, $\rho$ is the difference between the number of positive-norm and negative-norm states, although the definition of trace can be changed to make all states contribute positively.

We can now extract $\rho$ from $Z$ by contour integration. Treat $\tau$ and $\bar{\tau}$ as independent complex variables (this is not necessary, but it simplifies the computation), and let $q=e^{2 \pi i \tau}$ and $\bar{q}=e^{2 \pi i \bar{\tau}}$, so

$$
\rho(\Delta, \bar{\Delta})=\frac{1}{(2 \pi i)^{2}} \int \frac{d q}{q^{\Delta+1}} \frac{d \bar{q}}{\bar{q}^{\bar{\Delta}+1}} Z(q, \bar{q})
$$

For notational simplicity, I will suppress the $\bar{\tau}$ dependence, and restore it only at the end of the computation. The basic trick is to note that

$$
Z(\tau)=e^{\frac{2 \pi i c}{24} \tau} Z_{0}(\tau)
$$

and to use the modular invariance of $Z_{0}$ to rewrite the contour integral in a form suitable for a saddle point approximation:

$$
Z(\tau)=e^{\frac{2 \pi i c}{24} \tau} Z_{0}(-1 / \tau)=e^{\frac{2 \pi i c}{24} \tau} e^{\frac{2 \pi i c}{24} \frac{1}{\tau}} Z(-1 / \tau)
$$

and thus

$$
\rho(\Delta)=\int d \tau e^{-2 \pi i \Delta \tau} e^{\frac{2 \pi i c}{24} \tau} e^{\frac{2 \pi i c}{24} \frac{1}{\tau}} Z(-1 / \tau) .
$$

The key to a saddle point approximation is to separate the integrand into a rapidly varying phase and a slowly varying prefactor. Let us assume for the moment-we will 
have to check this - that $Z(-1 / \tau)$ varies slowly near the extremum of the phase. For large $\Delta$, the extremum of the exponent is then

$$
\tau \approx i \sqrt{c / 24 \Delta}
$$

Substituting (2.8) back into the integral, we obtain

$$
\rho(\Delta) \approx \exp \left\{2 \pi \sqrt{\frac{c \Delta}{6}}\right\} Z(i \infty)
$$

yielding the Cardy formula (1.2).

We must now check the saddle point approximation. From (2.3),

$$
Z(i / \epsilon)=\sum \rho(\Delta) e^{-2 \pi \Delta / \epsilon}
$$

If the lowest eigenvalue of $L_{0}$ is $\Delta_{0}=0$, then $Z(i / \epsilon)$ approaches a constant as $\epsilon \rightarrow 0$, and the saddle point approximation is good. But if $\Delta_{0} \neq 0$, the factor $Z(-1 / \tau)$ in (2.7) varies rapidly near the putative saddle point, and the approximation is not valid. This is easily corrected, however. Define

$$
\tilde{Z}(\tau)=\sum \rho(\Delta) e^{2 \pi i\left(\Delta-\Delta_{0}\right) \tau}=e^{-2 \pi i \Delta_{0} \tau} Z(\tau),
$$

which goes to a constant as $\tau \rightarrow i \infty$. Then the integral for $\rho$ becomes

$$
\rho(\Delta)=\int d \tau e^{-2 \pi i \Delta \tau} e^{-2 \pi i \Delta_{0} \frac{1}{\tau}} e^{\frac{2 \pi i c}{24} \tau} e^{\frac{2 \pi i c}{24} \frac{1}{\tau}} \tilde{Z}(-1 / \tau)
$$

For $\Delta$ large, this integral can be evaluated in a saddle point approximation, giving

$$
\rho(\Delta) \approx \exp \left\{2 \pi \sqrt{\frac{\left(c-24 \Delta_{0}\right) \Delta}{6}}\right\} \rho\left(\Delta_{0}\right)=\exp \left\{2 \pi \sqrt{\frac{c_{e f f} \Delta}{6}}\right\} \rho\left(\Delta_{0}\right)
$$

Equation (2.13) is the generalization of (1.2) to theories in which $\Delta_{0} \neq 0$.

At first sight, the assumption that $\Delta_{0}=0$ seems innocuous. But there is a well known conformal field theory for which this assumption fails, Liouville theory. The Liouville action contains a single (albeit interacting) scalar field, and canonical quantization gives standard creation and annihilation operators [37]. The density of states should thus behave like that of an ordinary scalar field: we should use equation (1.2) with $c=1$. On the other hand, the central charge $c_{\text {Liou }}$ in Liouville theory is determined by the coupling constants, and can chosen arbitrarily, so the naive Cardy formula can give an arbitrarily large density of states. The solution, as noted by Kutasov and Seiberg [38], 
is that the minimum value of $L_{0}$ is not zero for normalizable states in Liouville theory. Instead [39],

$$
\Delta_{0}=\frac{c_{\text {Liou }}-1}{24}
$$

and thus $c_{\text {eff }}=c_{\text {Liou }}-24 \Delta_{0}=1$ in (2.13), as expected from canonical quantization. This example is directly relevant to the BTZ black hole, since $(2+1)$-dimensional gravity induces a Liouville theory at spatial infinity [40,41], and the central charge (1.1) can be understood as arising from this Liouville theory.

Another example of an "effective central charge" will be useful later. Start with standard affine Lie algebrał

$$
\left[J_{m}^{a}, J_{n}^{b}\right]=i f^{a b}{ }_{c} J_{m+n}^{c}+k m g^{a b} \delta_{m+n, 0}
$$

with the usual affine Sugawara construction for the Virasoro generators,

$$
L_{n}=\frac{1}{2 k+Q} \sum_{p} g_{a b}: J_{p}^{a} J_{n-p}^{b}:,
$$

which satisfy the algebra (2.1). This theory has a central charge $c$ determined by the group, and its asymptotic density of states is given by equation (1.2). Now consider the deformed Virasoro algebra [42, 43] generated by

$$
\tilde{L}_{n}=L_{n}+i n \alpha_{a} J_{n}^{a}+\frac{k}{2} \alpha_{a} \alpha^{a} \delta_{n 0}
$$

It is easy to check that the $\tilde{L}_{n}$ again satisfy the Virasoro algebra (2.1), but with a new central charge

$$
\tilde{c}=c+12 k \alpha_{a} \alpha^{a} .
$$

But the redefinition (2.17) has not changed the Hilbert space, so the asymptotic behavior of the density of states should not be affected.

Again, the answer lies in the failure of the naive Cardy formula. The shift of $L_{0}$ means that the lowest eigenvalue of $\tilde{L}_{0}$ is no longer zero, but rather

$$
\tilde{\Delta}_{0}=\frac{k}{2} \alpha_{a} \alpha^{a}
$$

The effective central charge in (2.13) is thus $c_{\text {eff }}=\tilde{c}-12 k \alpha_{a} \alpha^{a}=c$, and the deformation (2.17) does not change the asymptotic density of states. Like the Liouville case, this

${ }^{*}$ I use the metric $g_{a b}=\operatorname{Tr} T_{a} T_{b}$ to raise and lower indices; this convention leads to an occasional factor of two difference with some expressions in the literature. In (2.16), $Q$ is defined by $f_{a b c} f^{a b d}=Q \delta_{c}^{d}$. 
example is directly relevant to the BTZ black hole: in Refs. [31] and [35], the central charge (1.1) is obtained by precisely such a shift.

It is perhaps worth emphasizing the peculiarity of equation (2.13) for models with $\Delta_{0}<0$. A negative value of $\Delta_{0}$ implies an increase in the asymptotic density of states: it is as if a model with $\Delta_{0}=-1$ had 24 extra bosonic oscillators. As we shall see in section 1 , the relative contributions to $c_{\text {eff }}$ from $c$ and $\Delta_{0}$ can depend on the choice of boundary conditions, and it is possible that the central charge in Strominger's approach to black hole entropy might come entirely from a large negative value of $\Delta_{0}$. It would be valuable to understand explicitly - perhaps in a simpler model - exactly what mechanism is responsible for the contribution of $\Delta_{0}$ to the density of states.

\section{Counting States: Combinatorics}

The preceding section dealt with the indirect counting of states via the partition function. In this section, I will discuss a more transparent counting procedure, based on the combinatorics of creation operators. Let us begin with a standard example, a single scalar field, whose creation and annihilation operators form an affine Lie algebra (2.15) for the group $\mathbf{R}$ of real numbers. We choose a vacuum such that

$$
J_{n}|0\rangle=0 \quad \text { for } n>0
$$

and create excited states by acting with creation operators $J_{-n}$. Since $\left[L_{0}, J_{-n}\right]=n J_{-n}$ and $L_{0}|0\rangle=\alpha_{0}|0\rangle$ for some constant $\alpha_{0}$,

$$
L_{0}\left(J_{-n_{1}} J_{-n_{2}} \ldots J_{-n_{m}}\right)|0\rangle=\left(\alpha_{0}+n_{1}+n_{2}+\ldots+n_{m}\right)|0\rangle .
$$

The number of states with $L_{0}=\Delta$ is thus simply the number of distinct ways the quantity $\Delta-\alpha_{0}$ can be written as a sum of integers. This is the famous partition function $p\left(\Delta-\alpha_{0}\right)$ of number theory, whose asymptotic behavior is 44]

$$
\ln p\left(\Delta-\alpha_{0}\right) \sim 2 \pi \sqrt{\Delta / 6}
$$

This behavior agrees, as it should, with the Cardy formula (1.2) for $c=1$.

Extensions of this result to more than one field appear frequently in the string theory literature. However, a more general form seems not to be widely known 45. Suppose we start with bosonic "creation operators" $\phi_{n}^{\left(M_{n}\right)}$, with conformal dimensions

$$
\left[L_{0}, \phi_{n}^{\left(M_{n}\right)}\right]=\beta n \phi_{n}^{\left(M_{n}\right)} .
$$

Here $\beta$ is a constant, and the index $M_{n}$ distinguishes fields with identical dimensions. Let $\gamma(n)$ denote the degeneracy at conformal dimension $\beta n$, i.e., $M_{n}=1, \ldots, \gamma(n)$. We 
allow $\gamma(n)$ to be zero for some values of $n$ - the conformal dimensions need not be equally spaced. Now, suppose that the asymptotic behavior of the sum of degeneracies is

$$
\sum_{n \leq x} \gamma(n) \sim K x^{u}
$$

for large $x$. Then the number of states with $L_{0}=\Delta$ can be shown to grow as

$$
\ln \rho(\Delta) \sim \frac{1}{u}[u+1]^{u /(u+1)}[K u \Gamma(u+2) \zeta(u+1)]^{1 /(u+1)}[\Delta / \beta]^{u /(u+1)} .
$$

For a scalar field, $\gamma(n)=1$ and hence $K=u=1$; it is then easily checked that (3.6) reproduces (3.3). For $D$ fields, $\gamma(n)=D$, and $K=D$; the effect is equivalent to the introduction of central charge $c=D$ in (1.2). But equation (3.6) is considerably more general. Consider, for example, a set of fields with conformal dimensions $j(j+1)$ and multiplicities $2 j+1$, i.e.,

$$
\gamma(n)= \begin{cases}2 j+1 & \text { if } n=j(j+1) \\ 0 & \text { otherwise }\end{cases}
$$

An easy computation shows that $K=u=1$, so the asymptotic density of states is again given by (3.3), even though the states are no longer evenly spaced. I will return to this example in section 6 .

For fermionic creation operators, no corresponding result exists in the literature, but the generalization of (3.6) is straightforward. The key observation is that the bosonic generating function $p(q)$ and the fermionic generating function $\tilde{p}(q)$, given by

$$
\begin{aligned}
& p(q)=\prod_{n=1}^{\infty}\left(1-q^{n}\right)^{-\gamma(n)}=\sum \rho(n) q^{n} \\
& \tilde{p}(q)=\prod_{n=1}^{\infty}\left(1+q^{n}\right)^{\gamma(n)}=\sum \tilde{\rho}(n) q^{n}
\end{aligned}
$$

satisfy $\tilde{p}(q)^{-1} p(q)=p\left(q^{2}\right)$, or equivalently,

$$
\sum_{m} \rho(m) \tilde{\rho}(n-2 m)=\rho(n) .
$$

Using this relation and equation (3.6), it is fairly easy to show that

$$
\ln \tilde{\rho}(\Delta) \sim \frac{1}{u}[u+1]^{u /(u+1)}\left[K u \Gamma(u+2) \zeta(u+1)\left(1-2^{-u}\right)\right]^{1 /(u+1)}[\Delta / \beta]^{u /(u+1)} .
$$

For $u=1$, in particular, the only difference between expressions (3.10) and (3.6) is an extra factor of $1 / \sqrt{2}$ in (3.10), corresponding to the well-known fact that a fermionic oscillator contributes a factor of $1 / 2$ to the central charge. 
Unlike the partition function methods of the preceding section, the combinatoric techniques described here explicitly display the states that contribute to the entropy. Unfortunately, there is a price to pay: we need to start with a much more concrete description of the vacuum and the operator content of the theory. Ultimately, however, some counting procedure like this will be necessary to complete our understanding of black hole entropy.

\section{Where Do the Black Hole Degrees of Freedom Live?}

In typical approaches to black hole statistical mechanics, the degrees of freedom associated with the entropy are assumed to live on or near the horizon. Strominger's derivation, on the other hand, is based on a central charge (1.1) that describes the asymptotic behavior at spatial infinity. Yet another suggestion, due to Martinec [12], is that the central charge is a result of "anomaly inflow" - the entropy comes from D-brane dynamics at the horizon, but the conformal anomaly is transported to spatial infinity by the coupling to bulk degrees of freedom.

To understand this issue better, it is helpful to review the derivation of the central charge in the Chern-Simons formulation of (2+1)-dimensional gravity. A careful canonical analysis has been given by Bañados [35] and by Bañados, Brotz, and Ortiz [31] (see also [46]), and I will not repeat it here, but will give a brief heuristic derivation of their results.

It is well known that diffeomorphisms in a Chern-Simons theory are equivalent on shell to field-dependent gauge transformations. Indeed, the Lie derivative of the gauge potential (or connection one-form) $A$ is

$$
\mathcal{L}_{\xi} A=d(\xi \cdot A)+\xi \cdot d A=D_{A}(\xi \cdot A)+\xi \cdot F
$$

where $D_{A}$ is the exterior gauge-covariant derivative, $F$ is the field strength, and the dot denotes contraction of a vector with the first index of a form. The Chern-Simons field equations tell us that $F=0$, and the remaining term in (4.1) may be recognized as a gauge transformation with an infinitesimal parameter $\epsilon^{a}=\xi^{\mu} A_{\mu}{ }^{a}$.

Now consider a slice at constant time with an $S^{1}$ boundary, which may be a black hole horizon or spatial infinity. Pick a radial coordinate $\rho$ such that the boundary is located at $\rho=\rho_{0}$, and choose a gauge condition

$$
A_{\rho}^{a}=\alpha^{a}
$$

near the boundary, where $\alpha^{a}$ is a fixed element of the Lie algebra. Up to possible quantum corrections, the generator of gauge transformations at the boundary is [35]

$$
\mathcal{G}[\epsilon]=-\frac{k}{2 \pi} \int_{S^{1}} \epsilon_{a} A_{\phi}^{a} d \phi,
$$


so by (4.1), the generator of diffeomorphisms is

$$
\mathcal{G}[\xi]=-\frac{k}{2 \pi} \int_{S^{1}}\left(\frac{1}{2} \xi^{\phi} g_{a b} A_{\phi}^{a} A_{\phi}^{b}+\xi^{\rho} g_{a b} \alpha^{a} A_{\phi}^{b}\right) d \phi .
$$

The factor of $1 / 2$ in the first term reflects the fact that both copies of $A_{\phi}$ contribute to the Poisson brackets: schematically, $\left\{\xi^{\phi}\left(A_{\phi}\right)^{2}, F\right\} \sim 2 \xi^{\phi} A_{\phi}\left\{A_{\phi}, F\right\}$. To preserve the gauge condition (4.2), we should take $\xi^{\rho}$ and $\xi^{\phi}$ to be independent of $\rho$.

Now, a Chern-Simons theory on a manifold with boundary induces a Wess-ZuminoWitten model on the boundary, with an affine Lie algebra that is essentially generated by the $A_{\phi}$. More precisely, if we write

$$
A_{\phi}{ }^{a}=\frac{1}{k} \sum_{n=-\infty}^{\infty} J_{n}^{a} e^{i n \phi},
$$

the currents $J_{n}^{a}$ will obey the algebra (2.15). The first term in (4.4) is thus closely related to the Virasoro generator (2.16), and if we can restrict $\xi^{\rho}$, we have a chance of recovering the Virasoro algebra (2.1).

In particular, Bañados observed that if we choose $\xi^{\rho}=-\partial_{\phi} \xi^{\phi}$, we recover an algebra with a central charge that, up to quantum corrections, is equal to the value (1.1) found by Brown and Henneaux. This should not be surprising in light of the model discussed at the end of section 2. For this choice of $\xi^{\rho}$, the generator (4.4) becomes

$$
\mathcal{G}[\xi]=-\frac{k}{2 \pi} \int_{S^{1}} \xi^{\phi}\left(\frac{1}{2} g_{a b} A_{\phi}{ }^{a} A_{\phi}{ }^{b}+\alpha_{a} \partial_{\phi} A_{\phi}{ }^{a}\right) d \phi,
$$

which leads to precisely the shift (2.17) of the Virasoro generators. Using the results from Appendix A that $\alpha_{a} \alpha^{a}=1 / 2$ and $k=\ell / 4 G$ for the BTZ black hole, we see that the shift in $c$ is $3 \ell / 2 G$, in agreement with (1.1).

But it is also clear that the generators (4.4) contain many other Virasoro subalgebras [31. If, for example, we choose $\xi^{\rho}=-\beta \partial_{\phi} \xi^{\phi}$, we obtain a central charge $c(\beta)=3 \beta^{2} \ell / 2 G$. We must somehow determine which choice gives us the "right" algebra; that is, we must decide what boundary conditions to place on the diffeomorphisms.

There are a number of natural choices, which are unfortunately not all equivalent. For example, we might fix the induced metric $g_{\phi \phi}$ on the boundary, by requiring that

$$
\mathcal{L}_{\xi} g_{\phi \phi}=0=\xi^{\rho} \partial_{\rho} g_{\phi \phi}+2 \partial_{\rho} \xi^{\phi} g_{\phi \phi},
$$

where $\rho$ is the proper radial coordinate (A.5). A simple calculation then shows that for a boundary at Schwarzschild coordinate $r=r_{0}$,

$$
\xi^{\rho}=-\frac{N(\infty)}{N\left(r_{0}\right)} \partial_{\phi} \xi^{\phi},
$$


where $N(r)$ is the BTZ lapse function (A.2). From equation (2.18), we thus obtain a central charge

$$
\tilde{c}\left(r_{0}\right)=c+\left(\frac{N(\infty)}{N\left(r_{0}\right)}\right)^{2} \frac{3 \ell}{2 G} .
$$

The naive Cardy formula (1.2) would thus give the standard BTZ entropy (1.4) for a boundary at infinity, but a "blue-shifted" entropy proportional to $N(\infty) / N\left(r_{0}\right)$ for a boundary at $r=r_{0}$. But this is precisely the kind of situation in which (1.2) is not to be trusted, since the minimum eigenvalue $\Delta_{0}$ of $L_{0}$ is also blue-shifted. Indeed, as we saw at the end of section 2, these shifts cancel in the effective central charge in the generalized Cardy formula (2.13), and the actual entropy is independent of $r_{0}$. Note, though, that we can no longer claim that this entropy is given by equation (1.4), unless we can control both $c$ and $\Delta_{0}$ at the boundary.

Rather than fixing the intrinsic geometry $g_{\phi \phi}$ at the boundary, we might equally plausibly fix the extrinsic curvature. For example, we could fix the radial form of York's "extrinsic time," $\Pi=\sqrt{g_{\phi \phi}} g^{\phi \phi} k_{\phi \phi}$, where $k_{\phi \phi}$ is the extrinsic curvature of our $S^{1}$ boundary viewed as a submanifold of a constant-time slice. Fixing $\Pi$ requires that

$$
\mathcal{L}_{\xi} \Pi=0=\partial_{\rho}\left(\xi^{\rho} \Pi\right)+\partial_{\phi}\left(\xi^{\phi} \Pi\right),
$$

and a straightforward computation shows that

$$
\xi^{\rho}=-\frac{N\left(r_{0}\right)}{\partial_{\rho} N\left(r_{0}\right)} \partial_{\phi} \xi^{\phi} .
$$

The corresponding central charge now varies from Strominger's value (1.1) at spatial infinity to zero at the horizon. Once again, however, the variation of $\Delta_{0}$ cancels this effect in the computation of the entropy.

As yet another alternative, we might choose to fix the mean curvature $k=g^{\phi \phi} k_{\phi \phi}$ at the boundary. Since $k$ is a scalar, this requires that

$$
\mathcal{L}_{\xi} k=0=\xi^{\rho} \partial_{\rho} k
$$

and thus $\xi^{\rho}=0$. This choice is physically appealing, since the condition for an apparent horizon on a time slice of vanishing mean curvature is that $k=0$ 47. On the other hand, such an apparent horizon is equally well determined by the condition that $\Pi=0$.

We can learn three basic lessons from this analysis:

1. At least in the Chern-Simons formulation of $(2+1)$-dimensional gravity, the central charge of an induced conformal field theory at a boundary can depend sensitively on the location of the boundary and the choice of boundary conditions. 
2. For the counting of states, this dependence may not matter, since the effective central charge in the generalized Cardy formula (2.13) may not change.

3. To use (2.13) to compute the entropy of the BTZ black hole, we must control not only the central charge, but also the eigenvalue $\Delta_{0}$, on some boundary.

\section{Lowest Virasoro Eigenvalues}

We have seen that if we wish to use the Cardy formula to compute the BTZ black hole entropy, we must know both the central charge and the lowest eigenvalue of $L_{0}$ on some boundary. Unfortunately, the general arguments of Brown and Henneaux [32] and Bañados [35] tell us little about the eigenvalue $\Delta_{0}$; for that, we need more information about the relevant conformal field theory.

One way to obtain such information comes from supersymmetry. As Coussaert and Henneaux have observed [48], the massless BTZ black hole is supersymmetric, and lies in the Ramond sector of the theory (i.e., the Killing spinors are periodic). Similarly, anti-de Sitter space - the " $M=-1 / 8 G$ " BTZ black hole -is supersymmetric, and lies in the Neveu-Schwarz sector (the Killing spinors are antiperiodic). Suppose the standard superconformal algebra,

$$
\begin{aligned}
{\left[L_{m}, L_{n}\right] } & =(m-n) L_{m+n}+\frac{c}{12} m\left(m^{2}-1\right) \delta_{m+n, 0} \\
{\left[L_{m}, G_{n}\right] } & =\left(\frac{1}{2} m-n\right) G_{m+n} \\
\left\{G_{m}, G_{n}\right\} & =2 L_{m+n}+\frac{c}{3}\left(m^{2}-\frac{1}{4}\right) \delta_{m+n, 0},
\end{aligned}
$$

applies to the boundary conformal field theory. In the Ramond sector, the generators $G_{m}$ have integer moding, and the lowest possible eigenvalue of $L_{0}$ will be

$$
L_{0}\left|0_{R}\right\rangle=\frac{c}{24}\left|0_{R}\right\rangle .
$$

In the Neveu-Schwarz sector, the $G_{m}$ have half-integer moding, and the lowest weight is

$$
L_{0}\left|0_{N S}\right\rangle=0 .
$$

The $M=0$ black hole thus has $L_{0}=c / 24$, and anti-de Sitter space has $L_{0}=0.0$

\footnotetext{
${ }^{\dagger}$ The $L_{0}$ values in Ref. 1 differ from these by an additive constant of $-c / 24$. This constant was chosen to adjust $L_{0}$ to vanish for the $M=0$ black hole. To use the Cardy formula for the density of states, however, we must normalize $L_{0}$ according to the algebra (5.1).
} 
If we can consider anti-de Sitter space to be part of our Hilbert space, and if the canonical analysis of Bañados in Ref. 35 can be extended to give the superconformal algebra (5.1), we can then argue that the lowest eigenvalue of $L_{0}$ is in fact $\Delta_{0}=0$. If this is the case, the generalized Cardy formula (2.13) reduces to (1.2), and Strominger's analysis gives the correct black hole entropy.

There is one subtlety in this argument, however. Although the $M=0$ BTZ black hole and anti-de Sitter space are certainly both supersymmetric, we do not know a priori which set of Virasoro generators $L_{n}$ appears in the superconformal algebra (5.1). As we saw in section 4, the canonical algebra of boundary diffeomorphisms, at the horizon or at infinity, contains many copies of the Virasoro algebra with different central charges, and we do not know which of these should be associated with anti-de Sitter space.

In particular, the deformation (2.17) of the Virasoro algebra of a WZW model can be extended to the supersymmetric case, with the same shift in central charge. In addition to the currents $J_{n}^{a}$, a supersymmetric WZW model contains a set of fermionic oscillators $\psi_{n}^{a}$ in the adjoint representation [49]. It is not hard to check that the deformation

$$
\widetilde{G}_{n}=G_{n}-2 i \sqrt{k} n \alpha_{a} \psi_{n}^{a}
$$

accompanied by the shift 2.17) of the $L_{n}$, gives a new superconformal algebra (5.1) with the shifted central charge (2.18). Note that in the Neveu-Schwarz sector, equation (5.4) shifts the operators $G_{ \pm 1 / 2}$, and the ambiguity can be reformulated as a question of which of these shifted operators annihilates the anti-de Sitter vacuum.

Supersymmetric Liouville theory again offers a cautionary tale. The super-Liouville model is a superconformal field theory, with an algebra (5.1) that can be constructed explicitly from the fields. It is tempting to conclude that supersymmetry should force the minimum eigenvalue of $L_{0}$ to be zero. But in fact, the stress-energy tensor of superLiouville theory contains an "improvement" term of the form (2.17), and $L_{0}$ is shifted by a constant 51

$$
\tilde{\Delta}_{0}=\frac{1}{24}\left(c-\frac{3}{2}\right)
$$

yielding an effective central charge of $c_{\text {eff }}=3 / 2$, as one would expect from counting oscillators. As in the nonsupersymmetric version [39], the candidate for an SL(2, C)invarant vacuum state is not normalizable, and does not lie in the Hilbert space built from the oscillators of the model.

Whether the same problem occurs for $(2+1)$-dimensional gravity can probably be determined only by a careful extension of a canonical analysis like that of Ref. 35, with close attention paid to the relationships between boundary conditions for the diffeomorphisms and their superpartners. An important step in this direction has recently been taken by Bañados et al. [52], who examine the asymptotic algebra of symmetries in $(2+1)$-dimensional supergravity and construct a superconformal algebra. I believe, 
however, that their description of the symmetry algebra is not yet explicit enough to determine the spectrum, and thus the eigenvalue $\tilde{\Delta}_{0}$ and the effective central charge.

I will end this section by pointing out a numerical coincidence that may have a deeper meaning. The Virasoro generator $L_{0}$ of equation (2.16) involves an important zero-mode term. For $\operatorname{SL}(2, \mathbf{R})$, with the conventions described in Appendix A, this contribution is

$$
L_{0}=\frac{1}{k-2}\left(-J_{0}^{2}+J_{1}^{2}+J_{2}^{2}\right)+\text { non-zero mode contributions, }
$$

where $J_{0}, J_{1}$, and $J_{2}$ obey the standard $\operatorname{SL}(2, \mathbf{R})$ commutation relations. From the representation theory of $\mathrm{SL}(2, \mathbf{R})$ [50], we see that for the principle discrete series,

$$
L_{0}=-\frac{j(j+1)}{k-2}+\text { non-zero mode contributions, }
$$

where $j$ is a negative integer or half-integer. In particular, for $j=-k / 2$,

$$
L_{0}=-\frac{k}{4}+\text { non-zero mode contributions }=-\tilde{\Delta}_{0}+\ldots,
$$

where $\tilde{\Delta}_{0}$ is the shift in the lowest eigenvalue of $L_{0}$, equation (2.19), for the BTZ black hole. The deformation (2.17) of $L_{0}$ thus precisely cancels the zero-mode contribution of the state with $j=-k / 2$.

Now, if the value $j=-k / 2$ had been chosen arbitrarily, this would not be a very significant observation. But in an SU(2) Chern-Simons theory, $j=k / 2$ is the highest admissible value (the highest integrable representation), and Hwang has argued that $j=-k / 2$ could play an equivalent role for $\operatorname{SL}(2, \mathbf{R})$ [53,54]. Similarly, in the Euclidean partition function approach of Ref. 30, $|j|=|k / 2|$ is the maximal value appearing in the partition function. This may be accidental, but it may indicate that the proper deformation (2.17) of the Virasoro algebra simultaneously sets the central charge to the value (1.1) and sets $\Delta_{0}$ to zero.

\section{Operators and Degrees of Freedom}

Suppose we can show that the "correct" central charge for the BTZ black hole at some boundary is given by equation (1.1), and that the corresponding lowest mode of $L_{0}$ is $\Delta_{0}=0$, so Strominger's derivation (1.2)-(1.4) is correct. We will still be left with a question: while the partition function tells us how many states there are, it does not in itself tell us what those states are. It was argued in Ref. [56] (see also [57]) that the relevant excitations are "would-be gauge" degrees of freedom, excitations that would

\footnotetext{
${ }^{\ddagger}$ See also 55]; note that $k$ in that reference is $k / 2$ in the conventions of this paper.
} 
normally be pure gauge, but that become physical as a result of boundary conditions. But this still does not explicitly express the excitations in terms of conformal field theory states. In this final section, I will speculate briefly on how we might obtain a more transparent description.

A possible starting point is Liouville theory, which provides a well-studied example of an "effective central charge" of the sort discussed in section 2. States in Liouville theory fall into two classes [39]: the normalizable "macroscopic" (or "anti-Seiberg") states, whose lowest Virasoro eigenvalue is given by (2.14), and the nonnormalizable "microscopic" (or "Seiberg," or "Hartle-Hawking") states, for which $\Delta=0$ can occur. The division reflects a breakdown of the usual operator-state correspondence of conformal field theory: insertions of local operators give nonnormalizable "microscopic" states. This example suggests that if we are looking for BTZ states with $\Delta_{0}=0$, we ought to investigate operator insertions rather than concentrating on the standard ("macroscopic") Hilbert space of oscillators.

Now, the fundamental operator in a Wess-Zumino-Witten model is not the current $J^{a}$, but the group-valued field $g$. The conformal weight of $g$ is not integral: for a spin- $j$ $\mathrm{SL}(2, \mathbf{R})$ representation in the principle discrete series,

$$
\Delta_{j}(g)=\frac{j(j-1)}{k-2}
$$

where $j$ is a positive integer or half-integer. The $\mathrm{SL}(2, \mathbf{R})$ WZW model is not yet understood well enough to determine which values of $j$ appear in the operator product expansion of $g$, but let us suppose that all do. We must further determine the multiplicities. This is also not known, but a reasonable guess is that the spin $j$ occurs with a multiplicity $2 j+1$, the Plancheral measure for the representation $j[54$.

(For a compact group $G$, the Peter-Weyl theorem tells us that any function $F(g)$ can be written as a sum over irreducible representations of $G$. The Plancheral formula is, roughly speaking, a generalization to noncompact groups, allowing $F(g)$ to be expressed as an integral over irreducible representations. Let $\widehat{G}$ be the space of isomorphism classes of unitary representations of $G$, with $U \in \widehat{G}$ a representation, and let

$$
\widehat{U}[F]=\int_{G} F(h) U(h) d h,
$$

where $d h$ is the Haar measure. Then the Plancheral formula tells us that

$$
F(g)=\int_{\widehat{G}} \operatorname{Tr}\left(\widehat{U}(F) U(g)^{*}\right) d \mu(U)
$$

\footnotetext{
§This is not obvious: for an $\mathrm{SU}(2) \mathrm{WZW}$ model, the representations with $j \geq k / 2$ completely decouple. But the null states for affine $\operatorname{SL}(2, \mathbf{R})$ are quite different from those for affine $\mathrm{SU}(2)$, so the analogy may be misleading. As Strominger has pointed out [58], one may also worry about whether the operators obtained in this fashion are mutually local (or, for that matter, whether such a constraint is necessary).
} 
where $d \mu(U)$ is the Plancheral measure [59]. This measure is thus a reasonable indication of how many times a given irreducible representation should be counted.)

We can now use equation (3.6) to compute the number of states that can be created by these operators, assuming that there are no relations among their products. From the discussion following equation (3.7), we see that the resulting entropy is

$$
\ln \rho(\Delta) \sim 2 \pi \sqrt{\frac{k \Delta}{3}} .
$$

Equation (6.4) almost argrees with Strominger's expression (1.1)-(1.2) for the BTZ black hole entropy. It differs by a factor of three inside the square root (or a factor

of six if we assume instead that each operator can appear only once and use (3.10) for the density of states). I do not know how to explain this factor; it may indicate that this approach to counting states fails. But it is also possible that the missing factor reflects the "bimodular" properties of the WZW model. As Chau and Yamanaka have stressed [60], the group-valued field $g$ in a WZW model has two independent transformation properties: it transforms on one side according to the standard $\mathrm{SL}(2, \mathbf{R})$ Lie algebra, and on the other side under an appropriate quantum group. It is plausible that the "extra" quantum group transformation properties lead to a further degeneracy in the number of states within a given representation of $\mathrm{SL}(2, \mathbf{R})$.

\section{Conclusion}

The derivation of BTZ black hole entropy in Ref. 4 seems too elegant to be wrong. Unfortunately, it is also too simple to be completely right: as we have seen-and as Strominger already noted in 4 - it involves assumptions about the relevant conformal field theory that are not obviously true for the black hole. It thus joins the previous derivations as a highly suggestive, but not quite complete, computation of black hole entropy from first principles.

With recent developments in higher-dimensional black hole entropy and anti-de Sitter "holography," the problem of giving a complete, explicit description of the degrees of freedom responsible for BTZ black hole entropy seems increasingly urgent. But the task is perhaps no longer hopelessly difficult. I will conclude this paper with a list of open questions. The answer to any one of these would represent progress in our knowledge of BTZ black hole entropy; answers to all would indicate a fairly solid understanding of the subject.

1. According to the generalized Cardy formula (2.13), a minimum Virasoro eigenvalue $\Delta_{0}<0$ leads to a drastic increase in the asymptotic growth of the density of states; a value $\Delta_{0}=-1$ has the same effect as 24 bosonic oscillators. Can this effect be understood explicitly in terms of a counting argument like those of section 3]? 
2. For an $\mathrm{SL}(2, \mathbf{R}) \mathrm{WZW}$ theory, zero modes can give negative contributions to $L_{0}$. Is there any reason to prefer the spin $j=-k / 2$, which would lead to a correction $-24 \Delta_{0}=6 k=3 \ell / 2 G$ in the effective central charge, agreeing with equation (1.1)?

3. Is there any natural way to choose among the boundary conditions for diffeomorphisms described in section 14 ?

4. Supersymmetry suggests a minimum Virasoro eigenvalue of $\Delta_{0}=0$, but the argument seems to fail for super-Liouville theory, presumably because the candidate for an $\operatorname{SL}(2, \mathbf{C})$-invariant vacuum is not a normalizable state. Does this problem extend to (2+1)-dimensional anti-de Sitter supergravity? Alternatively, are there other reasons to expect that $\Delta_{0}=0$ for some choice of boundary conditions consistent with the central charge (1.1)?

5. Does the breakdown of the operator-state relationship in Liouville theory [39] apply as well to the boundary conformal field theory induced from $(2+1)$-dimensional gravity? If so, is there a way to explicitly count the nonnormalizable ("microscopic") states?

6. The operator-counting approach of section $[$ is suggestive, but it depends on several uncertain assumptions (appearance of all values of $j$, multiplicities, independence and consistency of operators) and misses a factor of three in the final answer. Is there a way to make this argument more rigorous, or alternatively to demonstrate that it is incorrect?

7. The counting method of Ref. 27 differs from others in several respects. Some of the differences are discussed below in appendix Appendix B, but others - most notably involving the role of zero modes - remain mysterious. Can the connection between this method and that of Ref. 4 be understood more clearly?

8. Can the Euclidean partition function methods of Refs. 30 and 31 be related to Lorentzian state-counting methods? How are the states and zero modes mapped from one signature to the other?

\section{Appendix A Metrics, Coordinates, and Conventions}

The BTZ black hole is a solution to the vacuum Einstein equations in $2+1$ dimensions with a negative cosmological constant $\Lambda=-1 / \ell^{2}$. In Schwarzschild-like coordinates, the BTZ metric is [1]

$$
d s^{2}=-N^{2} d t^{2}+N^{-2} d r^{2}+r^{2}\left(d \phi+N^{\phi} d t\right)^{2}
$$


with lapse and shift functions

$$
N=\left(-8 G M+\frac{r^{2}}{\ell^{2}}+\frac{16 G^{2} J^{2}}{r^{2}}\right)^{1 / 2}, \quad N^{\phi}=-\frac{4 G J}{r^{2}} \quad(|J| \leq M \ell) .
$$

The outer (event) and inner horizons are located at

$$
r_{ \pm}^{2}=4 G M \ell^{2}\left\{1 \pm\left[1-\left(\frac{J}{M \ell}\right)^{2}\right]^{1 / 2}\right\}
$$

i.e.,

$$
M=\frac{r_{+}^{2}+r_{-}^{2}}{8 G \ell^{2}}, \quad J=\frac{r_{+} r_{-}}{4 G \ell} .
$$

The radial coordinate $r$ is adapted to the circular symmetry of the solution, and is characterized by the property that a circle of constant $r$ has a circumference $2 \pi r$. In the exterior region $r>r_{+}$, we can instead choose a proper radial coordinate $\rho$ and a dimensionless time coordinate $\tau$, defined by [35]

$$
r^{2}=r_{+}{ }^{2} \cosh ^{2} \rho-r_{-}{ }^{2} \sinh ^{2} \rho, \quad \tau=t / \ell .
$$

The metric then becomes

$$
d s^{2}=-\sinh ^{2} \rho\left(r_{+} d \tau-r_{-} d \phi\right)^{2}+\ell^{2} d \rho^{2}+\cosh ^{2} \rho\left(r_{-} d \tau-r_{+} d \phi\right)^{2} .
$$

Einstein gravity in $2+1$ dimensions with a negative cosmological constant can be reexpressed as a Chern-Simons theory for the group $\operatorname{SL}(2, \mathbf{R}) \times \operatorname{SL}(2, \mathbf{R})$ [23, 24], with gauge potentials (connection one-forms)

$$
A^{( \pm) a}=\omega^{a} \pm \frac{1}{\ell} e^{a}
$$

where $e^{a}=e_{\mu}{ }^{a} d x^{\mu}$ is the triad and $\omega^{a}=\frac{1}{2} \epsilon^{a b c} \omega_{\mu b c} d x^{\mu}$ is the spin connection. The EinsteinHilbert action becomes

$$
I_{\text {grav }}=I_{\mathrm{CS}}\left[A^{(-)}\right]-I_{\mathrm{CS}}\left[A^{(+)}\right]
$$

where

$$
I_{\mathrm{CS}}=\frac{k}{4 \pi} \int_{M} \operatorname{Tr}\left\{A \wedge d A+\frac{2}{3} A \wedge A \wedge A\right\},
$$

is the Chern-Simons action. The value of the coupling constant $k$ depends on the choice of representation and the definition of the trace in (A.9). With the choice [31]

$$
T_{0}=\frac{1}{2}\left(\begin{array}{cc}
0 & -1 \\
1 & 0
\end{array}\right), \quad T_{1}=\frac{1}{2}\left(\begin{array}{cc}
1 & 0 \\
0 & -1
\end{array}\right), \quad T_{2}=\frac{1}{2}\left(\begin{array}{ll}
0 & 1 \\
1 & 0
\end{array}\right),
$$


one finds that

$$
k=\frac{\ell}{4 G}
$$

With these conventions, the metric in the affine Lie algebra (2.15) is $g_{a b}=\frac{1}{2} \eta_{a b}$, and $Q=-4$ in equation (2.16). .

For the BTZ black hole in the coordinates (A.6), the connection one-forms are

$$
\begin{aligned}
& A^{( \pm) 0}= \pm \frac{r_{+} \mp r_{-}}{\ell} \sinh \rho(d \tau \pm d \phi) \\
& A^{( \pm) 1}= \pm d \rho \\
& A^{( \pm) 2}=\frac{r_{+} \mp r_{-}}{\ell} \cosh \rho(d \tau \pm d \phi) .
\end{aligned}
$$

From equation (4.2), $\alpha^{( \pm) a}= \pm \delta_{1}^{a}$, confirming that $\alpha_{a} \alpha^{a}=1 / 2$. The fields (A.12) can be converted by a simple gauge transformation to

$$
A^{( \pm)}=\frac{r_{+} \mp r_{-}}{\ell} T_{2}(d \tau \pm d \phi)
$$

from which the holonomies can be read off directly.

\section{Appendix B Polarizations}

The entropy calculation of Ref. 27 differs from others in two striking respects. First, while most approaches treat left- and right-movers independently, this reference lumps the left- and right-moving oscillators together and considers states created by both from a single vacuum. Second, the computation involves an integration over a zero mode $\bar{\omega}$ of the spin connection at the horizon. These two features are actually closely related: as I shall now show, both reflect the choice of boundary conditions or "polarization."

The partition function for the boundary degrees of freedom can be obtained as a path integral for $(2+1)$-dimensional gravity on a solid torus $M$, with appropriate boundary conditions on the fields at $\partial M \approx T^{2}$. Such a path integral may also be interpreted as determining a state on $\partial M$, viewed as a function of the boundary data. The choice of boundary conditions is thus equivalent to a choice of polarization, that is, of which phase space variables to treat as "positions" in the argument of the wave function.

In the approaches of Refs. 4 and 30, the boundary data are spatial components $A_{\phi}^{( \pm)}$ (or $A_{z}^{( \pm)}$) of the connection (A.7). In particular, the component $\omega_{\phi}=\left(A_{\phi}^{(+)}+A_{\phi}^{(-)}\right) / 2$ of the spin connection is fixed at the boundary. The corresponding partition functions are

$$
Z_{A}^{ \pm}=\sum \rho\left(N^{ \pm}\right) \exp \left\{2 \pi i \tau\left(\Delta^{ \pm}+N^{ \pm}\right)\right\}
$$

*I use the conventions of Ref. 31: $\eta_{a b}=\operatorname{diag}(-1,1,1)$ and $\epsilon_{012}=1$. 
where $\Delta^{ \pm}$are the zero modes of $L_{0}^{ \pm}$and

$$
N^{ \pm}=\sum_{i=1}^{3} N_{i}^{ \pm}
$$

are affine $\operatorname{SL}(2, \mathbf{R})$ number operators.

In Ref. 27, on the other hand, the boundary is fixed to be a null surface, with boundary datal $e_{r}{ }^{2}=r_{+} / \sqrt{2}$ and $e_{v}{ }^{2}=0$, where $v$ is a null coordinate. Now, $e_{v}{ }^{2}$ and $\omega_{\phi}^{2}$ are canonically conjugate, so the partition function in this new polarization can be obtained from (B.1) by a functional Fourier transformation (see, for example, [61]):

$$
Z_{e}=\int[d \omega] \exp \left\{\frac{2 i \tilde{k}}{\pi} \int \omega_{\phi}^{2} e_{v}^{2} d \phi\right\} Z_{A}^{+} Z_{A}^{-} .
$$

Since we are interested in the boundary condition $e_{v}{ }^{2}=0$, the exponential term in (B.3) drops out, and since only the zero mode of $\omega_{\phi}^{2}$ appears in $Z_{A}^{ \pm}$, only the integration over this mode is relevant. Thus

$$
Z_{e}=\sum_{N^{+}, N^{-}} \rho\left(N^{+}\right) \rho\left(N^{-}\right) e^{2 \pi i \tau\left(N^{+}+N^{-}\right)} \int d \bar{\omega} e^{2 \pi i \tau\left(\Delta^{+}+\Delta^{-}\right)} .
$$

Now, the conformal field theory of Ref. 27 describes excitations above a fixed black hole background, unlike that of [1], for instance, in which the vacuum is anti-de Sitter space. In particular, the black hole mass and angular momentum now determine the zero modes $\Delta^{ \pm}$, and the physical states are fixed by the condition that $L_{0}=0$ rather than by the relation (1.3). The integral over $\bar{\omega}$ is then exactly that of [27], and gives a factor of

$$
\exp \left\{-2 \pi i \tau\left(\frac{2 \tilde{k}^{2} r_{+}^{2}}{\ell^{2}}\right)\right\}
$$

in $Z_{e}$. If we now let $N^{+}+N^{-}=N$, the sum over $N^{-}$in (B.4) may be performed by the method of steepest descents. In particular, let $\rho\left(N^{ \pm}\right)$be determined by the counting arguments of section 3, with the three oscillators (B.2) in each sector:

$$
\rho\left(N^{ \pm}\right) \sim \exp \left\{2 \pi \sqrt{N^{ \pm} / 2}\right\}
$$

We then obtain

$$
\sum_{N^{-}} \rho\left(N^{-}\right) \rho\left(N-N^{-}\right) \sim \sum_{N^{-}} \exp \left\{\sqrt{2} \pi\left(\sqrt{N^{-}}+\sqrt{N-N^{-}}\right)\right\} \sim \exp \{2 \pi \sqrt{N}\} .
$$

${ }^{\dagger}$ Note that the choice of group generators in [27] differs from that used elsewhere in this paper, and that the coupling constant is therefore renormalized to $\tilde{k}=k / \sqrt{2}$. The superscript 2 in this section is a Lie algebra index. 
Combining these results, we find that

$$
Z_{e} \sim \sum_{N} \exp \{2 \pi \sqrt{N}\} \exp \left\{2 \pi i \tau\left(N-\frac{2 \tilde{k}^{2} r_{+}^{2}}{\ell^{2}}\right)\right\}
$$

The physical state condition $L_{0}=0$ thus requires that $N=2 \tilde{k}^{2} r_{+}^{2} / \ell^{2}$, and the density of states in (B.7) reproduces the Bekenstein entropy (1.4).

This derivation highlights another key difference between Ref. 27 and Strominger's approach, the use of the "naive" central charge $c \approx 3$ in (B.5) rather than the much larger central charge (1.1). This does not necessarily mean that the two derivations are incompatible - the change of polarization described here is, in part, a change of basis, and the counting of states can appear quite different in different bases. But it is clear that the zero modes, which are responsible for the first factor in (B.7), again play a crucial and rather mysterious role.

\section{Acknowledgements}

This paper reflects insights (and occasional arguments) that have come from conversations with a number of people, including Max Bañados, Ling-Lie Chau, Marty Halpern, Marc Henneaux, Stephen Hwang, Miguel Ortiz, and Andy Strominger. This work was supported in part by National Science Foundation grant PHY-93-57203 and Department of Energy grant DE-FG03-91ER40674.

\section{References}

[1] M. Bañados, C. Teitelboim, and J. Zanelli, Phys. Rev. Lett. 69, 1849 (1992).

[2] M. Bañados, M. Henneaux, C. Teitelboim, and J. Zanelli, Phys. Rev. D48, 1506 (1993).

[3] S. Carlip, Class. Quant. Grav. 12, 2853 (1995).

[4] A. Strominger, hep-th/9712251, J. High Energy Phys. 02, 009 (1998).

[5] D. Birmingham, I. Sachs, and S. Sen, Phys. Lett. B424, 275 (1998).

[6] V. Balasubramanian and F. Larsen, Nucl. Phys. B528, 229 (1998).

[7] M. Iofa and L. A. Pando Zayas, "Statistical Entropy of Magnetic Black Holes from Near-Horizon Geometry," hep-th/9803083. 
[8] S. P. de Alwis, "Supergravity, the DBI Action and Black Hole Physics," hepth/9804019.

[9] N. Kaloper, "Entropy Count for Extremal Three-Dimensional Black Strings," hepth/9804062.

[10] J. Maldacena and A. Strominger, " $A d S_{3}$ Black Holes and a Stringy Exclusion Principle," hep-th/9804085.

[11] H. W. Lee and Y. S. Myung, "Holographic Connection between the BTZ Black Hole and 5D Black Hole," hep-th/9804095.

[12] E. J. Martinec, "Matrix Models of AdS Gravity," hep-th/9804111.

[13] M. Iofa and L. A. Pando Zayas, "Statistical Entropy of Calabi-Yau Black Holes," hep-th/9804129.

[14] K. Behrndt, I. Brunner, and I. Gaida, Phys. Lett. B432, 310 (1998).

[15] I. Sachs, "On Universality in Black Hole Thermodynamics," hep-th/9804173.

[16] E. Teo, "Black Hole Absorption Cross-Sections and the anti-de Sitter-Conformal Field Theory Correspondence," hep-th/9805014.

[17] H. W. Lee, N. J. Kim, and Y. S. Myung, "Dilaton Test of Holography between $A d S_{3} \times S^{3}$ and 5D Black Hole," hep-th/9805050.

[18] M. Cvetič and F. Larsen, "Near Horizon Geometry of Rotating Black Holes in Five Dimensions," hep-th/9805097.

[19] M. Cvetič and F. Larsen, "Microstates of Four-Dimensional Rotating Black Holes from Near-Horizon Geometry," hep-th/9805146.

[20] S. Hyun, "U-Duality between Three-Dimensional and Higher Dimensional Black Holes," hep-th/9704005.

[21] K. Sfetsos and K. Skenderis, Nucl. Phys. B517, 179 (1998).

[22] E. Teo, Phys. Lett. B430, 57 (1998).

[23] A. Achúcarro and P. K. Townsend, Phys. Lett. B180, 89 (1986).

[24] E. Witten, Nucl. Phys. B311, 46 (1988).

[25] E. Witten, Commun. Math. Phys. 121, 351 (1989). 
[26] S. Elitzur et al., Nucl. Phys. B326, 108 (1989).

[27] S. Carlip, Phys. Rev. D51, 632 (1995).

[28] M. Bañados and A. Gomberoff, Phys. Rev. D55, 6162 (1997).

[29] A. Strominger and J. Maldacena, gr-qc/9801096, J. High Energy Phys. 02, 014 (1998).

[30] S. Carlip, Phys. Rev. D55, 878 (1997).

[31] M. Bañados, T. Brotz, and M. E. Ortiz, "Boundary Dynamics and the Statistical Mechanics of the 2+1 Dimensional Black Hole," hep-th/9802076.

[32] J. D. Brown and M. Henneaux, Commun. Math. Phys. 104, 207 (1986).

[33] J. A. Cardy, Nucl. Phys. B270, 186 (1986).

[34] H. W. J. Blöte, J. A. Cardy, and M. P. Nightingale, Phys. Rev. Lett. 56, 742 (1986).

[35] M. Bañados, Phys. Rev. D52, 5816 (1995).

[36] A. Giveon, D. Kutasov, and N. Seiberg, "Comments on String Theory on AdS $S_{3}$," hep-th/9806194.

[37] T. L. Curtright and C. B. Thorn, Phys. Rev. Lett. 48, 1309 (1982); Ann. Phys. (N.Y.) 147, 365 (1983).

[38] D. Kutasov and N. Seiberg, Nucl. Phys. B358, 600 (1991).

[39] N. Seiberg, Prog. Theor. Phys. Suppl. 102, 319 (1990).

[40] S. Carlip, Nucl. Phys. B362, 111 (1991).

[41] O. Coussaert, M. Henneaux, and P. van Driel, Class. Quant. Grav. 12, 2961 (1995).

[42] J. K. Freericks and M. B. Halpern, Ann. Phys. (N.Y.) 188, 258 (1988).

[43] N. Sakai and P. Suranyi, Nucl. Phys. B362, 655 (1989).

[44] S. Ramanujan and G. H. Hardy, Proc. London Math. Soc. (ser. 2) 17, 75 (1918), reprinted in Collected Papers of Srinivase Ramanujan, edited by G. H. Hardy et al. (Chelsea Publishing Company, NY, 1962).

[45] N. A. Brigham, Proc. Amer. Math. Soc. 1, 182 (1950). 
[46] P. Oh and M.-I. Park, "Symplectic Reduction and Symmetry Algebra in Boundary Chern-Simons Theory," hep-th/9805178.

[47] A. R. Steif, Phys. Rev. D53, 5527 (1996).

[48] O. Coussaert and M. Henneaux, Phys. Rev. Lett. 72, 183 (1994).

[49] E. B. Kiritsis and G. Siopsis, Phys. Lett. B184, 353 (1987).

[50] See, for example, A. Inomata, in A. Inomata, H. Kuratsuji, and C. C. Gerry, Path Integrals and Coherent States of $S U(2)$ and $S U(1,1)$ (World Scientific, Singapore, 1992).

[51] H. C. Liao and P. Mansfield, Nucl. Phys. B344, 696 (1990).

[52] M. Bañados, K. Bautier, O. Coussaert, M. Henneaux, and M. E. Ortiz, "Anti-de Sitter/CFT Correspondence in Three-Dimensional Supergravity," hep-th/9805165.

[53] S. Hwang, Nucl. Phys. B354, 100 (1991).

[54] S. Hwang and P. Roberts, in Pathways to Fundamental Theories, Proc. of the 16th Johns Hopkins Workshop on Current Problems in Particle Theory, edited by L. Brink and R. Marnelius (World Scientific, Singapore, 1993).

[55] J. M. Evans, M. R. Gaberdiel, and M. J. Perry, "The No-Ghost Theorem for $A d S_{3}$ and the Stringy Exclusion Principle," hep-th/9806024.

[56] S. Carlip, in Contrained Dynamics and Quantum Gravity 1996, edited by V. de Alfaro et al., Nucl. Phys. B (Proc. Suppl.) 57, 8 (1997).

[57] A. P. Balachandran, L. Chandar, and A. Momen, Nucl. Phys. B461, 581 (1996).

[58] A. Strominger, personal communication.

[59] S. Lang, $S L_{2}(R)$ (Springer, New York, 1985).

[60] L.-L. Chau and I. Yamanaka, Phys. Lett. B369, 226 (1996).

[61] H. Verlinde, Nucl. Phys. B337, 652 (1990). 